\title{
Der Fünfliber oder Soziale Marktwirtschaft: Win-win auf Kosten von wem?
}

Markus Gassner

Korrespondenz:

Dr. med. Markus Gassner

Spitalstr. 8

CH-9472 Grabs

m.gassner[at]hin.ch
Viele Menschen machen sich Sorgen um ihr Geld, manche aus Armut, andere aus Gier. Wenige aber schauen ihr Geld an und hinterfragen den Sinn der Darstellungen (auf den Zahlungsmitteln) oder dessen Zweck. In der internationalen Finanzmarktregulierung ist der Ursprung und die Bedeutung des Geldes für ein Individuum kaum mehr erkennbar. Kurz nach Beginn der letzten Weltwirtschaftskrise fragte die Königin Elisabeth II. anlässlich der Einweihung eines 71 Millionen teuren Erweiterungsbau der London School of Economics am 5.11.2008 die Anwesenden: Weshalb hat keiner von Ihnen die Krise vorhergesehen? [1].

Geld wurde und wird zwar in unterschiedlichen Kulturen benützt: zum Tausch von Gütern und Dienstleistungen wurden Steine, Münzen, Noten, lokale Wechselpapierchen eingesetzt. Bei grossen Wertverschiebungen in der Kapitalmarktregulierung spielt das Geld an sich aber kaum mehr eine Rolle. Hier geht es um die Regulation von Krediten. Kredit bedeutet ursprünglich Glauben, Vertrauen (credere). Ursprünglich hatte zwar ein «Gläubiger» einem vertrauenswürdigen Menschen Geld zum Arbeiten gegeben oder eine Schuld übernommen. Über den Handel und das Anhäufen von Krediten und Schulden entstand ein Kapital, das letztlich einem Sammelsurium von «Wertpapieren» entspricht. Solange alle an ihren Kredit glauben, bleibt alles friedlich. Eine Krise entsteht, wenn ein grosser oder viele kleine ihren Kredit zurückfordern und damit die Liquidität einer staatlichen oder privaten Institution (Bank) existenziell herausfordern. Meist wird dies verursacht durch den Verlust der Glaubwürdigkeit dieser Institution.

\section{«Kredit bedeutet ursprünglich Glauben, Vertrauen (credere).»}

\section{Der Fünfliber}

Es geht hier nicht um den wörtlichen Sinn der Bezeichnung unserer grössten Münze, die eigentlich «fünf Pfund» bedeutet. Fünf Pfund herumzutragen ist unpraktisch. Weniger Volumen war praktisch für den Geldbeutel.

Auf dem Fünfliber ist ein Mann abgebildet. Viele halten ihn irrtümlich für Wilhelm Tell, der als Schiffer am Vierwaldstättersee gelebt haben soll. Knapp sichtbar auf der Münze ist der Name des Künstlers Paul Burkhard (1888-1964). Als Bildhauer und Zeichner hatte er 1919-1921 am Wettbewerb für die 2- und 5-Franken-Stücke teilgenommen und durfte dann das Münzbild für den Fünfliber «Alphirte» prägen lassen. Der ursprünglich eingereichte Alphirte, auf einem Felsen stehend oder mit Schweizer Fahne, wurde dann als Portrait in Auftrag gegeben [2]. Auf dem Fünfliber ist also nicht Wilhelm Tell zu sehen, sondern ein namenloser Alphirte.

Wichtiger als das Abbild sind die vier lateinischen Wörter, die auf dieser Münze geprägt sind. Sie mögen auf den Sinn und Zweck des Geldes zum Abfedern von Gewinnschwankungen oder eigener Gewinn hinweisen.

\section{Geld als konservierte Arbeit}

Zentral ist die stets aktuelle Frage nach dem Sinn des Geldes überhaupt. Manche Völker verwendeten Geld in Form einer Münze, zum Tausch eine Ware oder Dienstleistung. Für die Akzeptanz der unterschiedlichsten Zahlungsmittel war aber stets das Vertrauen auf dessen inneren Wert massgebend. Geld entsprach der konservierten Arbeit. Der Besitzer von Münzen konnte damit Saatgut kaufen oder ein Haus bauen. Für den Staat wurde es einfacher, Steuern in Form von Münzen einzutreiben, als den «Zehnten» Teil der Ernte. Selbst ein Tyrann kann nicht so viel Ochsen, Fastnachtshühner oder Wein selbst verzehren oder damit Soldaten ernähren, damit diese noch mehr Land für noch mehr Zehnten erobern. Dies hat bisher gemäss der Geschichte auf der ganzen Welt nur vorübergehend funktioniert.

Geld als konservierte Arbeit wird von vielen Ökonomen abgelehnt. Managerlöhne lassen sich damit schwer rechtfertigen. Geld sei deshalb konservierte 


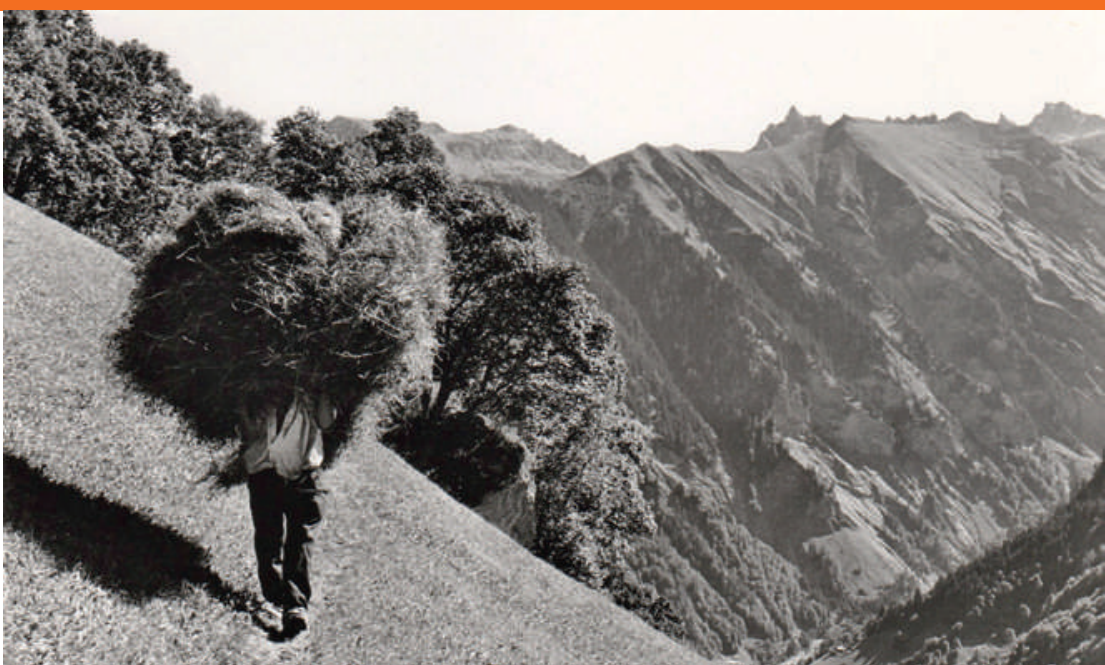

So produzierte dieser Bergbauer in Oberrüti ob Weisstannen (Sarganserland) Nahrungsmittel. Seine Vorfahren hatten das Land «gerodet». Trotz Agrikultur ein mühsames, gefährliches Leben. - Wie viel kostet ein Liter seiner Milch? Dieses Land wird heute vom Wald zurückerobert. (Foto: Pius Rupf, Flums [3])

Leistung. Physikalisch bedeutet Leistung die in einer Zeiteinheit verrichtete Arbeit. An der Arbeitszeit kann es dabei nicht liegen, denn jeder Mensch verfügt über genau gleich viel Zeit. Der Wert einer Idee in einem Moment, ein Gedankenblitz als üblicher Ursprung einer Kreativität ist so nicht messbar, ökonomisch irrelevant, sonst wären Künstler und Erfinder die reichsten Menschen, oder die Reichsten müssten am meisten eigene originelle, produktive und nachhaltige Ideen produzieren. Wer zertifiziert Manager nach welchen Kriterien? Auch der Energieverbrauch ist kaum relevant, denn dieser ist bei geistiger Arbeit kleiner.

\section{Geld und die konservierte Kultur}

Der Alphirte auf der Münze zeigt die älteste Kultur, die ein Überleben unserer Bevölkerung ermöglichte, die Agrikultur. Die Abbildung zeigt den Bergbauern Simi Schneider aus Weisstannen (1912-2003) bei der Heuernte, wie sie hier bis in dieses Jahrhundert durchgeführt wurde. Sie war die Voraussetzung für das Überwintern der Kühe, die über die Käseverarbeitung (Kunst der Milchkonservierung) den Handel und das Überleben der Bauern ermöglichte.

Was aber kostet ein Liter Milch, produziert von diesem Mann wie auf dem Fünfliber oder im vollautomatisierten Stall mit Kraftfutter aus importierten Sojabohnen? - Was kostet welche Laborbestimmung, Operation, ein Hausbesuch, wann und wo, z. B. bei diesem Bauern? Wer darf welche Leistung auf Kosten von wem erbringen, zu welchem Taxpunktwert? Somit stellt sich die berechtigte Frage nach dem, was Leistungen ermöglicht.

Daraus ergibt sich die Bedeutung der Tradition, unser kulturelles Erbe: das Wissen und die Erfahrung, wie man Werkzeuge macht, womit man Werke erzeugen kann sowie die Bildung und die Infrastruktur. All das wird heute so ganz selbstverständlich zur Geldproduktion vorausgesetzt, konsumiert. Aber nicht jeder hat die gleichen Möglichkeiten, gleich viel davon zu benutzen, z. B. Menschen in den Bergen, anderswo, Kranke, Personen mit geringer Bildung usw. sche Aussenhandelsstatistik Schweiz Ärztezeitung. 1990; 71:603-6.

\section{Zum Wert der Heilkunde}

Wie die Agrikultur zur Ernährung pflegt auch jedes Volk die Heilkunde. Kranke Menschen sind meist nicht leistungsfähig. Invalid übersetzt heisst «wertlos», für die Familie und den Staat eine Belastung. Für Versicherungen sind sie ein Schaden oder nicht versicherbar. Absolut sicher ist nur der Tod. Nur Unsicherheiten sind versicherbar, die Dauer und die Qualität des Lebens. Unsere staatliche «Confoederatio» ist für eine optimale Gesundheit aller Einwohner verantwortlich. Deshalb erwarten ihre «Genossenschafter» von uns Ärzten, dass wir uns um die Gesundheit und das Leben aller einsetzen. Jeder Patient ist eine lebendige Persönlichkeit, als solche nicht normierbar. Die Kultur und die Kunst der Heilkunde hat aber nicht die Aufgabe, die Rendite einer Versicherung zu verbessern. Kriterien der gesunden Prämienzahler sind für manche moderne Gesundheitsökonomen das Wichtigste. Damit lasse sich Gesundheit, Pflege marktwirtschaftlich automatisch regeln. Das Schweizer Volk hatte hierzu seine berechtigten Zweifel und hat am 17. Juni 2012 immerhin mit einer Mehrheit von 76\% «Managed Care» abgelehnt. Unser Souverän möchte die Pflege nicht so regeln. Heute spürt man kaum etwas von diesem Volkswillen. Die Spitäler werden über ein DRG AG Konzept verwaltet, was vor allem neue Probleme schafft. Krankenkassen haben sich so weit vermarktwirtschaftlicht, entsolidarisiert, dass eine Einheitskasse notwendig wird. Win-win im Sozialbereich muss äusserst sorgfältig hinterfragt werden, auf Kosten von wem: Solidarität bedeutet ein grösseres «win» für die Schwächeren!

\section{Zur Kultur im Wert des Geldes}

Das Wissen über die Geschichte, die Kultur und die Infrastruktur haben unsere Vorfahren mit Steuern und Abgaben finanziert. Unsere Aufgabe ist es, diese Werte unseren Kindern und Enkelkindern weiterzugeben. Manche Produkte und Dienstleistungen, auch Personen lassen sich importieren und exportieren. Die entsprechenden Werte von Dingen in Franken und Kilo kann man in der Schweizerischen Aussenhandelsstatistik nachschauen [4]. Soziale Probleme sind nicht exportierbar (z. B. Pflege von kranken und alten Einwohnern). Darf ein Spital rentieren - auf Kosten von anderen Spitälern - oder was?

Man könnte auch nachdenken, wozu wir arbeiten! Welche Dinge, welche Dienstleistung, welche Arbeit (Lohn) sind uns wie viel wert: Eine 1.-AugustRakete oder eine Zeitung, ein Buch, eine Wellness«Kur» oder eine Impfung. Wer soll welche Vorsorge, Forschung, welche Sicherheit mit welchem Geld für wen bezahlen? Wie konservieren wir welche Werte? Welche Lasten, ungelösten Probleme überlassen wir der nächsten Generation?

- Für was sind uns, dem Staat $(\mathrm{CH})$ wie viele Fünfliber wert?

- Soziale Marktwirtschaft: Win-win auf Kosten von wem? 